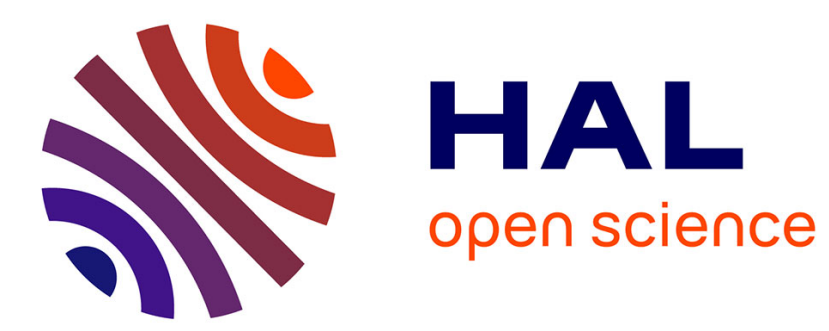

\title{
Prediction of flow induced sound and vibration of periodically stiffened plates
}

\author{
Laurent Maxit, Vivien Denis
}

\section{To cite this version:}

Laurent Maxit, Vivien Denis. Prediction of flow induced sound and vibration of periodically stiffened plates. Journal of the Acoustical Society of America, 2013, 133 (1), pp.146-160. 10.1121/1.4768875 . hal-00879256

\section{HAL Id: hal-00879256 \\ https://hal.science/hal-00879256}

Submitted on 2 Nov 2013

HAL is a multi-disciplinary open access archive for the deposit and dissemination of scientific research documents, whether they are published or not. The documents may come from teaching and research institutions in France or abroad, or from public or private research centers.
L'archive ouverte pluridisciplinaire HAL, est destinée au dépôt et à la diffusion de documents scientifiques de niveau recherche, publiés ou non, émanant des établissements d'enseignement et de recherche français ou étrangers, des laboratoires publics ou privés. 
Prediction of flow induced sound and vibration of periodically stiffened plates

\author{
Laurent Maxit ${ }^{\mathrm{a}}$ \\ Laboratoire Vibrations-Acoustique (LVA), \\ INSA Lyon, \\ 25 bis av. Jean Capelle, \\ 69621 Villeurbanne Cedex France \\ Vivien Denis \\ Laboratoire d'Acoustique de l'Universite du Maine (LAUM), \\ av. Olivier Messiaen, \\ 72085 Le Mans Cedex 9 - France
}

(Dated: September 24, 2012)

Response to turbulent boundary layer 1 


\begin{abstract}
Stiffened structures excited by the Turbulent Boundary Layer (TBL) occur very frequently in engineering applications, for instance in the wings of airplanes or the pressure hulls of submarines. To improve knowledge of the interaction between stiffened structures and TBL, this paper deals with the modelling of infinite periodically stiffened plates excited by TBL. The mathematical formulation of the problem is wellestablished in the literature. The originality of the present work relies on the use of a wavenumber-point reciprocity technique for evaluating the response of the plate to convected harmonic pressure waves. It follows a methodology for estimating the vibro-acoustic response of the plate excited by the TBL from the wall pressure spectrum and its displacements in the wavenumber space due to point excitations located at the receiving positions. The computing process can be reduced to the numerical integration of an analytical expression in the case of a periodically stiffened plate. An application to a naval test case highlights the effect of Bloch-Floquet waves on the vibrations of the plate and its radiated pressure in the fluid.
\end{abstract}

PACS numbers: 43.40.Dx, 43.40.Rj 


\section{INTRODUCTION}

Stiffened structures excited by the turbulent boundary layer (TBL) are very common in practical applications. Airplanes, trains and submarines are all composed of stiffened structures that are excited by pressure fluctuations due to the turbulent flow induced by their movement. In order to reduce the noise radiated from these structures, it is important to understand how a stiffened structure reacts to TBL excitation. In particular, some phenomena related to periodically stiffened panels, such as the well known pass bands and stop bands, have an influence on the radiated noise of these structures when they are excited by mechanical forces $[1,2]$. It is logical to ask whether these phenomena are equally important for TBL excitation. To improve knowledge in this domain, the present paper proposes a model of infinite periodically stiffened plates excited by TBL.

There are many works in the literature on simple plates excited by a turbulent flow and mention can be made of the review of existing models of finite and infinite plates under turbulence by Strawderman (1969) [3]. Although neither the finite nor infinite model agrees wholly with the experimental results, he indicated that the vibration statistics computed from the finite plate model are in better agreement with the experimental results than those computed from the infinite panel model (especially for the cross-spectral properties). He also investigated [4] the effect of heavy fluid loading on the vibratory response for finite and infinite plates under the turbulent boundary layer. He noticed that for heavy fluid, the inertial component of the fluid loading is several orders of magnitude greater than the resulting component. As for a light fluid, he noted that the infinite plate velocity spectral density provides a good approximation of the finite plate velocity spectral density, but that substantial differences occur on the cross-spectral characteristics. Davis (1971) [5] proposed a space integration method including the light fluid loading effect to estimate the power density functions of the displacement of the finite plate and of the radiated acoustic pressure. Comparisons with experimental results showed that the modal predictions were slightly bet-

\footnotetext{
a)Electronic address: laurent.maxit@insa-lyon.fr
} 
ter that those obtained with the Statistical Energy Analysis method. Aupperle and Lambert (1972) [6] studied the acoustic radiation from finite rectangular plates excited by flow noise and concluded that the radiated power was dependent on the low-wavenumber spectrum of the excitation below the hydrodynamic coincidence frequency. Rumerman (1992) [7] studied the excitation mechanisms of a simply supported plate under TBL excitation. He obtained an expression for the one-third octave spatially averaged mean square acceleration of the plate due to excitation by TBL pressure. Graham (1995) [8] reviewed statistical models of the boundary layer and investigated the more specific case of aircraft statistical models of TBL. The response of a finite panel under boundary layer excitation was determined by using the modal superposition method and a wavenumber integration technique. For naval applications, Ko and Schloemer (1987) [9] proposed a method for evaluating the transmitted flow noise received by a rectangular hydrophone embedded in an infinite extended viscoelastic layer. The wavenumber filtering effets of, both the elastomer layer and the rectangular hydrophone were highlighted by their approach. The technique was extended to an array of finite hydrophones embedded in a viscoelastic layer in Ref. [10]. More recently, Maury et al. (2002) [11] proposed a self-contained and general description of a wavenumber approach to modelling the response of a randomly excited panel. This was in line with the wavevectorfrequency analysis of the different authors reviewed by Strawderman in 1988 [12] and this formulation gives a physical interpretation of the interaction between the turbulent flow and the dynamic behaviour of the panel, especially in terms of a filtering effect of the structure in the wavenumber domain. Mazzoni (2002) [13] proposed a deterministic model to approximate the response of an elastic rectangular plate at a low Mach number. The approximation was based on the observations of numerical studies showing that the subconvective region of the turbulent excitation power spectrum contributes significantly to the response of the panel.

In addition to these studies dealing with turbulence-induced plate vibrations and radiated noise, many authors have studied the vibroacoustic behaviour of periodically stiffened structures. A review of the works carried out at the University of Southampton can be found 
in Ref. [14]. Concerning the periodically stiffened plates considered in this study, Rumerman (1974) [15], Mace (1980) [1] and Maidanik and Dickey (1991) [16] proposed similar formalisms for studying the vibrations and radiated sound of these plates. Rumerman [15] and Mace [1] focused on the propagation of free waves and the forced vibration of a ribbed plate due to the harmonic pressure field. Mace also derived expressions for the stiffened plate responses due to point and line force excitations and discussed the effect of fluid loading, noting the presence of acoustically damped propagating waves in the plate due to radiation losses. Maidanik and Dickey [16] studied the reflection of incident acoustic plane waves by ribbed panels. They observed that the phenomenon of pass and stop bands played a role in the reflection properties of ribbed, fluid-loaded panels.

Although many studies have been devoted to stiffened structures, few of them have focused on stiffened structures excited by the turbulent boundary layer. Mention can be made however of the study proposed by Rumerman (2001) [17]. He derived expressions giving broad band (i.e. one-third octave) estimations of the acoustic power radiated from a finite ribbed plate excited by TBL. He assumed a wavenumber-white pressure excitation and that the ribs radiated independently. Requiring these two assumptions, the method appeared to be more accurate in the high frequency domain.

In this paper, we propose an alternative method to that proposed by Rumerman, without taking into account the two assumptions mentioned above. The use of the wavenumberfrequency formalism for evaluating the response of a panel randomly excited in time and space is well-established in the literature. Strawderman [12] has presented a general description of this approach used recently by Maury [11] and Aucejo [18] for airplane and naval applications, respectively. This formalism permits estimating the random response from knowledge of the wavenumber-frequency spectrum of the excited pressure and from the responses of a panel for a set of harmonic plane waves. The present study is based on the same formalism. The originality of the present work relies on the use of a non classical reciprocity technique to calculate the plate response due to the harmonic plane waves. Indeed, point to point reciprocity [19] makes it possible to establish a reciprocity relation between the point 
response of the plate excited by harmonic plane waves and the response in the wavenumber space of the plate excited by a point source. This non standard reciprocity relation will be called the wavenumber-point reciprocity relation, or more concisely, the $(\mathrm{k}, \mathrm{M})$ reciprocity relation. As for a periodically stiffened plate immersed in a fluid, the plate displacements in the wavenumber space for point excitations can be calculated analytically [2], while the plate response to TBL excitation can be obtained easily with the $(\mathrm{k}, \mathrm{M})$ reciprocity relation. The calculation process is reduced to a numerical integration of an analytical expression. The computing time is relatively short and the present approach allows analysing the filtering effect of the excitation by the stiffened plate. This analysis can also be performed for the vibrations of the plate as its pressure radiated in the fluid. It will be discussed for a naval test case.

This paper is organized as follows:

- Section II consists in describing the outlines of the wavenumber-frequency formulation of the panel response excited by random pressure fields [12]. The (k,M) reciprocity technique is then introduced in order to facilitate the evaluation of the quantities characterizing the stiffened plates in this formalism;

- The modelling of the periodically stiffened plate loaded by a fluid in the wavenumber space is given in section III. Two point excitations are considered: A normal mechanical force on the plate and an acoustic monopole source in the fluid. These two cases permit evaluating both the vibrations and the radiated pressure from the plate excited by a TBL;

- Section IV gives more details on the numerical integration performed on the analytical expressions obtained in the previous sections;

- The results of the present approach are proposed and discussed in section $\mathrm{V}$ for a naval test case. The effect of Bloch-floquet wave propagation will be highlighted. 


\section{ESTIMATION OF THE SPACE FREQUENCY SPECTRUM OF THE PLATE RESPONSE DUE TO TURBULENT FLOW}

\section{A. Wavenumber-frequency formulation}

Here we use the outlines of the formulation described in the literature $[2,12,16]$. Let us consider a baffle panel of surface $\Sigma_{p}$ excited by a TBL as shown in figure 1 . Three hypotheses are considered. First, the TBL is assumed to be fully developed, stationary and homogeneous. Second, the plate and the boundary layer are weakly coupled, in other words the vibration of the plate does not interfere with the wall pressure. Third, it is assumed that the propagation of the acoustic waves in the fluid is not affected by the flow.

$p_{b}(\mathbf{x}, t)$ represents the wall pressure exerted by the TBL on the plate at point $\mathbf{x}$ as a function of time. The displacement of the plate on point $\mathbf{x}$ due to wall pressure $p_{b}$ can be expressed as the convolution product

$$
w(\mathbf{x}, t)=\int_{\Sigma_{p}} \int_{-\infty}^{+\infty} h_{w}(\mathbf{x}, \tilde{\mathbf{x}}, t-\tilde{\tau}) p_{b}(\tilde{\mathbf{x}}, \tilde{\tau}) \mathrm{d} \tilde{\tau} \mathrm{d} \tilde{\mathbf{x}}
$$

where $h_{w}(\mathbf{x}, \tilde{\mathbf{x}}, t)$ is the impulse response in the displacement at point $\mathbf{x}$ for a normal unit force at point $\tilde{\mathbf{x}}$.

Considering that the random process is ergodic, the inter-correlation function $R_{w w}\left(\mathbf{x}, \mathbf{x}^{\prime}, t\right)$ can be defined as

$$
R_{w w}\left(\mathbf{x}, \mathbf{x}^{\prime}, t\right)=\int_{-\infty}^{+\infty} w(\mathbf{x}, \tau) w\left(\mathbf{x}^{\prime}, t+\tau\right) d \tau
$$

When Eq. (1) is substituted in Eq. (2), we obtain

$$
\begin{aligned}
R_{w w}\left(\mathbf{x}, \mathbf{x}^{\prime}, t\right)=\iint_{\Sigma_{p}} \int_{-\infty}^{+\infty} \int_{-\infty}^{+\infty} \int_{-\infty}^{+\infty} h_{w}(\mathbf{x}, \tilde{\mathbf{x}}, \tau-\tilde{\tau}) \\
\quad \times p_{b}(\tilde{\mathbf{x}}, \tilde{\tau}) p_{b}(\tilde{\tilde{\mathbf{x}}}, \tilde{\tilde{\tau}}) h_{w}\left(\mathbf{x}^{\prime}, \tilde{\tilde{\mathbf{x}}}, t+\tau-\tilde{\tilde{\tau}}\right) \mathrm{d} \tilde{\tau} \mathrm{d} \tilde{\tilde{\tau}} \mathrm{d} \tau \mathrm{d} \tilde{\mathbf{x}} \mathrm{d} \tilde{\tilde{\mathbf{x}}} .
\end{aligned}
$$

One can rewrite this expression by considering the variable change $(\tilde{\tau}, \tilde{\tilde{\tau}}) \rightarrow(\tilde{\beta}, \tilde{\tilde{\beta}})$, 
$\tilde{\beta}=\tau-\tilde{\tau}, \tilde{\tilde{\beta}}=\tau-\tilde{\tilde{\tau}}$ with the associated Jacobian equal to one,

$$
\begin{aligned}
R_{w w}\left(\mathbf{x}, \mathbf{x}^{\prime}, t\right)=\iint_{\Sigma_{p}} & \int_{-\infty}^{+\infty} \int_{-\infty}^{+\infty} h_{w}(\mathbf{x}, \tilde{\mathbf{x}}, \tilde{\beta}) \\
& \times\left(\int_{-\infty}^{+\infty} p_{b}(\tilde{\mathbf{x}}, \tau-\tilde{\beta}) p_{b}(\tilde{\tilde{\mathbf{x}}}, \tau-\tilde{\tilde{\beta}}) \mathrm{d} \tau\right) h_{w}\left(\mathbf{x}^{\prime}, \tilde{\tilde{x}}, t+\tilde{\tilde{\beta}}\right) \mathrm{d} \tilde{\beta} \mathrm{d} \tilde{\tilde{\beta}} \mathrm{d} \tilde{\mathbf{x}} \mathrm{d} \tilde{\tilde{\mathbf{x}}} .
\end{aligned}
$$

In this expression, one can identify an integral corresponding to the inter-correlation function of the wall pressure between the points $\tilde{\mathbf{x}}$ and $\tilde{\tilde{\mathbf{x}}}, R_{p_{b} p_{b}}$,

$$
\int_{-\infty}^{+\infty} p_{b}(\tilde{\mathbf{x}}, \tau-\tilde{\beta}) p_{b}(\tilde{\tilde{\mathbf{x}}}, \tau-\tilde{\tilde{\beta}}) \mathrm{d} \tau=\int_{-\infty}^{+\infty} p_{b}(\tilde{\mathbf{x}}, \bar{\tau}) p_{b}(\tilde{\tilde{\mathbf{x}}}, \bar{\tau}+\tilde{\beta}-\tilde{\tilde{\beta}}) \mathrm{d} \bar{\tau}=R_{p_{b} p_{b}}(\tilde{\mathbf{x}}, \tilde{\tilde{\mathbf{x}}}, \tilde{\beta}-\tilde{\tilde{\beta}})
$$

Introducing Eq. (5) in Eq. (4) leads to

$$
R_{w w}\left(\mathbf{x}, \mathbf{x}^{\prime}, t\right)=\iint_{\Sigma_{p}} \iint_{-\infty}^{+\infty} h_{w}(\mathbf{x}, \tilde{\mathbf{x}}, \tilde{\beta}) R_{p_{b} p_{b}}(\tilde{\mathbf{x}}, \tilde{\tilde{\mathbf{x}}}, \tilde{\beta}-\tilde{\tilde{\beta}}) h_{w}\left(\mathbf{x}^{\prime}, \tilde{\tilde{\mathbf{x}}}, t+\tilde{\tilde{\beta}}\right) \mathrm{d} \tilde{\beta} \mathrm{d} \tilde{\tilde{\beta}} \mathrm{d} \tilde{\mathbf{x}} \mathrm{d} \tilde{\mathbf{x}}
$$

The space-frequency spectrum $S_{w w}\left(\mathbf{x}, \mathbf{x}^{\prime}, \omega\right)$ is obtained by taking the temporal Fourier transform of the inter-correlation function and can be written as

$$
S_{w w}\left(\mathbf{x}, \mathbf{x}^{\prime}, \omega\right)=\iint_{\Sigma_{p}} H_{w}^{*}(\mathbf{x}, \tilde{\mathbf{x}}, \omega) S_{p p}(\tilde{\mathbf{x}}, \tilde{\tilde{\mathbf{x}}}, \omega) H_{w}\left(\mathbf{x}^{\prime}, \tilde{\tilde{\mathbf{x}}}, \omega\right) \mathrm{d} \tilde{\mathbf{x}} \mathrm{d} \tilde{\tilde{\mathbf{x}}}
$$

where $H_{w}(\mathbf{x}, \tilde{\mathbf{x}}, \omega)$ is the transfer function in displacement of the structure observed on point $\mathbf{x}$, driven by a point force at point $\tilde{\mathbf{x}}$, and $S_{p p}(\tilde{\mathbf{x}}, \tilde{\tilde{\mathbf{x}}}, \omega)$ is the space-frequency spectrum of the wall pressure (i.e. the Fourier transform of $R_{p_{b} p_{b}}$ ).

Defining the wavenumber-frequency spectrum of the wall pressure $\phi_{p p}(\mathbf{k}, \omega)$ as the space Fourier transform of $S_{p p}(\tilde{\mathbf{x}}, \tilde{\tilde{\mathbf{x}}}, \omega)$,

$$
S_{p p}(\tilde{\mathbf{x}}, \tilde{\tilde{\mathbf{x}}}, \omega)=\frac{1}{(2 \pi)^{2}} \iint_{-\infty}^{+\infty} \phi_{p p}(\mathbf{k}, \omega) e^{-j \mathbf{k}(\tilde{\tilde{\mathbf{x}}}-\tilde{\mathbf{x}})} \mathrm{d}^{2} \mathbf{k}
$$

one can write

$$
\begin{aligned}
S_{w w}\left(\mathbf{x}, \mathbf{x}^{\prime}, \omega\right)=\frac{1}{(2 \pi)^{2}} \iint_{-\infty}^{+\infty} \phi_{p p}(\mathbf{k}, \omega) & \\
& \times \iint_{S_{p}} H_{w}^{*}(\mathbf{x}, \tilde{\mathbf{x}}, \omega) e^{j \mathbf{k} \tilde{\mathbf{x}}} \mathrm{d} \tilde{\mathbf{x}} \\
& \times \iint_{S_{p}} H_{w}\left(\mathbf{x}^{\prime}, \tilde{\tilde{\mathbf{x}}}, \omega\right) e^{-j \mathbf{k} \tilde{\mathbf{x}}} \mathrm{d} \tilde{\tilde{\mathbf{x}}} \mathrm{d}^{2} \mathbf{k} .
\end{aligned}
$$


In this expression, the space Fourier transforms of $H_{w}^{*}(\mathbf{x}, \tilde{\mathbf{x}}, \omega)$ and $H_{w}\left(\mathbf{x}^{\prime}, \tilde{\tilde{\mathbf{x}}}, \omega\right)$ appear:

$$
\tilde{H}_{w}^{\omega}(\mathbf{x}, \mathbf{k})=\iint_{S_{p}} H_{w}(\mathbf{x}, \tilde{\mathbf{x}}, \omega) e^{j \mathbf{k} \tilde{\mathbf{x}}} \mathrm{d} \tilde{\mathbf{x}}
$$

$\tilde{H}_{w}^{\omega}(\mathbf{x}, \mathbf{k})$ is the frequency response observed at point $\mathbf{x}$ of the plate excited by a plane wave of wavevector $\mathbf{k}$. Indeed it can be interpreted as the sum of the responses at point $\mathbf{x}$ of the plate due to excitations of amplitude $e^{j \mathbf{k} \tilde{\mathbf{x}}}$ at points $\tilde{\mathbf{x}}$.

We can finally write the space-frequency spectrum of displacement, depending on the transfer function $\tilde{H}_{w}^{\omega *}(\mathbf{x}, \mathbf{k})$ and wall-pressure spectrum $\phi_{p p}(\mathbf{k}, \omega)$,

$$
S_{w w}\left(\mathbf{x}, \mathbf{x}^{\prime}, \omega\right)=\frac{1}{4 \pi^{2}} \iint_{-\infty}^{+\infty} \tilde{H}_{w}^{\omega *}(\mathbf{x}, \mathbf{k}) \phi_{p p}(\mathbf{k}, \omega) \tilde{H}_{w}^{\omega}\left(\mathbf{x}^{\prime}, \mathbf{k}\right) \mathrm{d}^{2} \mathbf{k}
$$

where $\mathbf{k}=\left[k_{x}, k_{y}\right], k_{x}$ is the wavenumber in the streamwise direction, and $k_{y}$ the wavenumber in the spanwise direction. If $\mathbf{x}$ and $\tilde{\mathbf{x}}$ are the same point, the spectral power density of the displacement at point $\mathbf{x}$ is

$$
S_{w w}(\mathbf{x}, \omega)=\frac{1}{4 \pi^{2}} \iint_{-\infty}^{+\infty}\left|\tilde{H}_{w}^{\omega}(\mathbf{x}, \mathbf{k})\right|^{2} \phi_{p p}(\mathbf{k}, \omega) \mathrm{d}^{2} \mathbf{k}
$$

Using the same approach the spectral power density of the pressure at point $\mathbf{z}$ in the fluid is given by Eq. (13)

$$
\begin{aligned}
& S_{p p}(\mathbf{z}, \omega)=\frac{1}{4 \pi^{2}} \iint_{-\infty}^{+\infty}\left|\tilde{H}_{p}^{\omega}(\mathbf{z}, \mathbf{k})\right|^{2} \phi_{p p}(\mathbf{k}, \omega) \mathrm{d}^{2} \mathbf{k} \\
& \text { with } \quad \tilde{H}_{p}^{\omega}(\mathbf{z}, \mathbf{k})=\iint_{\Sigma_{p}} H_{p}(\mathbf{z}, \tilde{\mathbf{x}}, \omega) e^{j \mathbf{k} \tilde{\mathbf{x}}} \mathrm{d} \tilde{\mathbf{x}}
\end{aligned}
$$

where $\tilde{H}_{p}^{\omega}(\mathbf{z}, \mathbf{k})$ represents the pressure at point $\mathbf{z}$ in the fluid when the plate is excited by a plane wave of wavevector $\mathbf{k}$.

In the following, it is assumed that the wavenumber-frequency spectrum of the wall pressure $\phi_{p p}(\mathbf{k}, \omega)$ is known. This implies the use of a model proposed in the literature, such as Corcos' [20] or Chase's [21] and that the TBL parameters (i.e. TBL thickness, convective and friction velocities) are known. 


\section{B. The $(k, M)$ reciprocity principle}

Expressions (12) and (13) allow estimating the vibrations of the plate and its radiated pressure in the fluid from knowledge of the wall pressure spectrum, $\Phi_{p p}$, and the transfer functions, $\tilde{H}_{w}^{\omega}$ and $\tilde{H}_{p}^{\omega}$. These transfer functions can be obtained by calculating the response of the panel excited by a harmonic plane wave of wavevector $\mathbf{k}$. To circumvent the continuous integration over the wavenumber space in Eq. (12) and Eq. (13), it is possible to truncate and sample the wavenumber space using appropriate criteria. The transfer functions $\tilde{H}_{w}^{\omega}$ and $\tilde{H}_{p}^{\omega}$ must then be estimated for a set of wavevectors. Computation time can become prohibitive as the number of wavevectors to be considered can be large. In this paper, to overcome this obstacle, we propose using the reciprocity principle [19]. This principle indicates that the ratio of the normal displacement of the plate at point $\mathbf{x}$ over the applied normal force at point $\mathbf{x}^{\prime}$ is equal to the ratio of the normal displacement of the plate at point $\mathbf{x}^{\prime}$ over the normal force applied at point $\mathbf{x}$. With the present notation, we can write

$$
H_{w}(\mathbf{x}, \tilde{\mathbf{x}}, \omega)=H_{w}(\tilde{\mathbf{x}}, \mathbf{x}, \omega)
$$

By introducing this expression in Eq. (10), we obtain

$$
\tilde{H}_{w}^{\omega}(\mathbf{x}, \mathbf{k})=\iint_{S_{p}} H_{w}(\tilde{\mathbf{x}}, \mathbf{x}, \omega) e^{j \mathbf{k} \tilde{\mathbf{x}}} \mathrm{d} \tilde{\mathbf{x}}
$$

which states that $\tilde{H}_{w}^{\omega}(\mathbf{x}, \mathbf{k})$ can also be interpreted as the space Fourier transform of the plate excited at point $\mathbf{x}$. Here, the two equivalent interpretations of $\tilde{H}_{w}^{\omega}(\mathbf{x}, \mathbf{k})$ are called the wavenumber-point $(\mathrm{k}, \mathrm{M})$ reciprocity principle. Consequently, the power spectrum density of the displacement of the plate at point $\mathbf{x}$ excited by the TBL can be calculated with Eq. (12) on the basis of the response of the plate excited by a normal force at point $\mathbf{x}$. That is to say that the plate response at a given point due to TBL can be estimated from the vibratory field of the plate excited by a point force at the same point. On the other hand, if the point of observation is in the fluid domain, the reciprocity principle (see Ref. [22, 23]), illustrated in figure 2, indicates that the ratio of the sound pressure at point $\mathbf{z}$ over the applied normal force at point $\mathbf{x}$ is equal to the ratio of the normal displacement of the plate 
at point $\mathbf{x}$ over the volume acceleration of the acoustic source located at point $\mathbf{z}$. The transfer function $\tilde{H}_{p}^{\omega}(\mathbf{z}, \mathbf{k})$ can therefore be interpreted as the space Fourier transform of the normal displacement of the plate for the volume acceleration of an acoustic source located at point $\mathbf{z}$. The $(\mathrm{k}, \mathrm{M})$ reciprocity still holds for a point $\mathrm{M}$ inside the fluid medium. The power spectral density of the pressure radiated by the plate at point $\mathbf{z}$ when the plate is excited by the TBL can be calculated with Eq. (13) from the knowledge of the response of the plate excited by an acoustic source located at point $\mathbf{z}$. This view, using the so-called $(\mathrm{k}, \mathrm{M})$ reciprocity principle, allows us to save computing time compared to the direct approach consisting in calculating the plate response for harmonic plane waves. Indeed, for the system considered in this paper (i.e. periodically stiffened plate immersed in a fluid), the displacement of the plate expressed in the wavenumber domain can be obtained analytically, as will be recalled in the next section. The computation time is therefore very short.

\section{WAVENUMBER SPACE RESPONSE OF THE RIBBED PLATE IMMERSED IN A FLUID}

Let us consider an infinite thin plate of thickness $h$ lying in the plane $z=0$ and with identical stiffeners. The stiffeners are assumed to be straight and uniform. They are attached along lines $x=n d, n$ being an integer and $d$, the distance between two stiffeners. The connection between the plate and the stiffeners is assumed to be rigid. The materials are linearly elastic, homogeneous and isotropic. The plate is loaded with an acoustic fluid on one side and is excited by a harmonic pressure while $F(x, y)$ represents the spatial distribution of this pressure. The harmonic time dependence $e^{j \omega t}$ is omitted in the following developments.

Under these assumptions, the normal displacement $W$ of the plate satisfies the LoveKirchhoff equation of motion (see Ref. [1, 24])

$$
\begin{aligned}
D^{*} \nabla^{4} W(x, y)-\omega^{2} \rho h W(x, y) & =F(x, y)-p(x, y, 0) \\
& -\sum_{n \in Z} F_{s}(x, y) \delta(x-n d)+\sum_{n \in Z} \frac{\partial}{\partial x}\left(M_{s}(x, y) \delta(x-n d)\right)
\end{aligned}
$$


where $F_{s}(x, y) \delta(x-n d)$ and $M_{s}(x, y) \delta(x-n d)$ are the force and moment distributions due to the interaction between the plate and the nth stiffener; $p(x, y, 0)$ is the wall pressure; $\rho, h, D^{*}$ are the mass density, the thickness and the flexural rigidity, respectively.

For beam-like stiffeners, the force and moment due to the nth stiffener can be written as (see Ref. $[1,24])$

$$
\begin{array}{r}
F_{s}(x, y)=E_{p}^{*} I_{x} \frac{\partial^{4} W}{\partial y^{4}}(x, y)-\omega^{2} \rho_{p} S_{p} W(x, y) \\
M_{s}(x, y)=-G_{p}^{*} J_{p} \frac{\partial^{3} W}{\partial x \partial y^{2}}(x, y)-\omega^{2} \rho_{p} I_{0} \frac{\partial W}{\partial x}(x, y)
\end{array}
$$

where $E_{p}^{*}, G_{p}^{*}, \rho_{p}, S_{p}$ are Young's and Coulomb's moduli, the density and the cross section of the stiffener, $I_{x}$ and $I_{0}$ are the moments of inertia, and $J_{p}$ is the torsion constant. Structural damping is introduced into the plate (respectively, stiffeners) material by assigning a complex value with a loss factor $\eta$ (respectively, $\eta_{p}$ ) to the elastic modulus.

The acoustic pressure $p(x, y, z)$ satisfies:

- the Helmholtz equation in the half space $\Omega_{p}$ occupied by the fluid,

$$
\nabla^{2} p(x, y, z)+k_{0}^{2} p(x, y, z)=0 \forall(x, y, z) \in \Omega_{p}
$$

with $k_{0}$ the acoustic wavenumber and $\nabla$ the Nabla operator,

- the Euler equation at the coupling area between the plate and the fluid, $\Sigma_{p}$,

$$
-\rho_{0} \omega^{2} W(x, y)=-\frac{\partial p}{\partial z}(x, y, 0)
$$

- the Sommerfeld radiation conditions on a fictional infinite surface, $\Sigma_{\infty}$.

Two independent excitations should be considered in order to satisfy the requirements of section II.B: A normal force on the plate and an acoustic monopole in the fluid.

For a normal force at point $\mathbf{x}=\left(x_{0}, 0\right)$, we have

$$
F(x, y)=P_{0} \delta\left(x-x_{0}\right) \delta(y)
$$

where $P_{0}$ represents the amplitudes of the excitation. In this case, $p(x, y, z)$ represents the total pressure at point $(x, y, z)$. 
Conversely, for an acoustic source at point $\mathbf{z}=\left(0,0, z_{0}\right)$, one has $F(x, y)=$ $P_{0}\left(e^{j k_{0} \sqrt{x^{2}+y^{2}+z_{0}^{2}}}\right) /\left(2 \pi \sqrt{x^{2}+y^{2}+z_{0}^{2}}\right)$ which represents the blocked pressure exerted on the plate due to the monopole source. In this case, $p(x, y, z)$ represents the pressure radiated by the plate at point $(x, y, z)$ (see Ref. [25] for details).

The problem described in this section can be solved in the wavenumber space. These developments have already been treated by Mace [1] and Maxit [2] for a point force excitation. Here it is extended for a monopole source, using the same process. This part is described in the appendix.

The developments in the wavenumber space $\mathbf{k}=\left(k_{x}, k_{y}\right)$ lead to an analytical expression of the plate displacement

$$
\begin{aligned}
\tilde{W}\left(k_{x}, k_{y}\right)=\frac{P_{0}}{Z\left(k_{x}, k_{y}\right)}[ & \Lambda\left(k_{x}, k_{y}\right) \\
& \quad-\frac{Z_{p}\left(k_{y}\right)\left(T_{0}\left(1+S_{2} A_{p}\left(k_{y}\right)\right)-T_{1} S_{1} A_{p}\left(k_{y}\right)\right)}{\Delta} \\
& \left.\quad-\frac{k_{x} A_{p}\left(k_{y}\right)\left(T_{0} S_{1} Z_{p}\left(k_{y}\right)-T_{1}\left(1+S_{0} Z_{p}\left(k_{y}\right)\right)\right)}{\Delta}\right]
\end{aligned}
$$

with

$$
\Delta=\left(\left(1+S_{0} Z_{p}\left(k_{y}\right)\right)\left(1+S_{2} A_{p}\left(k_{y}\right)\right)-S_{1}^{2} Z_{p}\left(k_{y}\right) A_{p}\left(k_{y}\right)\right)
$$

where $Z$ and $Z_{p}$ represent the flexural impedances of the plate and the stiffeners (see Eq. (A5) and (A6) in appendix), respectively, $A_{p}$ is the torsional impedance of the stiffeners (see Eq. (A7) in appendix), $S_{p}$ and $T_{p}$ are such as

$$
\left\{\begin{array}{l}
S_{p}=\sum_{n \in Z} \frac{\left(k_{x}+\frac{2 \pi n}{d}\right)^{p}}{Z\left(k_{x}+\frac{2 \pi n}{d}, k_{y}\right)} \\
T_{p}=\sum_{n \in Z} \frac{\left(k_{x}+\frac{2 \pi n}{d}\right)^{p} \Lambda}{Z\left(k_{x}+\frac{2 \pi n}{d}, k_{y}\right)}
\end{array}\right.
$$

and $\Lambda$ is given by

$$
\Lambda\left(k_{x}, k_{y}\right)= \begin{cases}e^{-j k_{x} x_{0}} & \text { for a force excitation at } \mathbf{x}=\left(x_{0}, 0\right), \\ \frac{e^{-k_{z} z_{0}}}{-k_{z}} & \text { for a monopole source at } \mathbf{z}=\left(0,0, z_{0}\right) .\end{cases}
$$

The quantity $\tilde{H}_{w}^{\omega}(\mathbf{x}, \mathbf{k})$ mentioned in the previous section corresponds to $\tilde{W}\left(k_{x}, k_{y}\right)$ obtained with expression (21) for the case of a point force at $\mathbf{x}=\left(x_{0}, 0\right)$ and the angular 
frequency $\omega$. These displacements are noted $\tilde{W}_{F\left(x_{0}\right)}\left(k_{x}, k_{y}, \omega\right)$. Similarly, $\tilde{H}_{p}^{\omega}(\mathbf{z}, \mathbf{k})$ corresponds to $\tilde{W}\left(k_{x}, k_{y}\right)$ for the case of a monopole source at $\mathbf{z}=\left(0,0, z_{0}\right)$ and the angular frequency $\omega$. These displacements are noted $\tilde{W}_{M\left(z_{0}\right)}\left(k_{x}, k_{y}, \omega\right)$.

\section{NUMERICAL INTEGRATION}

Now we consider the periodically stiffened plate excited by the TBL. The direction of the flow is perpendicular to stiffener direction y (i.e. along the x-axis). Eq. (12) and (13) permit estimating the response of the plate excited by the TBL from the wall pressure wavenumberfrequency spectrum and the displacement responses of the plate in the wavenumber space for the cases of a point force and a monopole source. In the previous section, analytical expressions were obtained for the latter quantities whereas different models (i.e. Corcos [20], Chase [21], Smol'yakov-Tkachenko [26], etc.) give expressions for the wall pressure in the wavenumber space. The integrands of Eq. (12) and (13) are fully defined. However, the integrals of these quantities cannot be calculated. Thus we suggest estimating these integrals numerically by truncating, sampling the wavenumber spaces and using the rectangular rule. A rectangular window centered on the origin of the wavenumber space is used for windowing. $\overline{k_{x}}, \overline{k_{y}}$ are the cutoff wavenumbers of the window and sampling is performed with the wavenumber resolutions, $\delta k_{x}, \delta k_{y}$ about $k_{x}$ and $k_{y}$ axis, respectively. The numerical integrations of Eq. (12) and (13) are written as

$$
\begin{aligned}
& S_{w w}\left(x_{0}, \omega\right) \approx \frac{\delta k_{x} \delta k_{y}}{4 \pi^{2}} \sum_{i=-N_{k_{x}}}^{N_{k_{x}}} \sum_{j=-N_{k_{y}}}^{N_{k_{y}}}\left|\tilde{W}_{F\left(x_{0}\right)}\left(k_{x}^{i}, k_{y}^{j}, \omega\right)\right|^{2} \times \phi_{p p}\left(k_{x}^{i}, k_{y}^{j}, \omega\right) \\
& S_{p p}\left(x_{0}, \omega\right) \approx \frac{\delta k_{x} \delta k_{y}}{4 \pi^{2}} \sum_{i=-N_{k_{x}}}^{N_{k_{x}}} \sum_{j=-N_{k_{y}}}^{N_{k_{y}}}\left|\tilde{W}_{M\left(z_{0}\right)}\left(k_{x}^{i}, k_{y}^{j}, \omega\right)\right|^{2} \times \phi_{p p}\left(k_{x}^{i}, k_{y}^{j}, \omega\right)
\end{aligned}
$$

where:

- $\tilde{W}_{F\left(x_{0}\right)}\left(k_{x}^{i}, k_{y}^{j}, \omega\right)$ is given by Eq. (21) in the case of a unit point force at $\left(x_{0}, 0\right)$,

- $\tilde{W}_{M\left(z_{0}\right)}\left(k_{x}^{i}, k_{y}^{j}, \omega\right)$ is given by Eq. (21) in the case of a unit monopole source at $\left(0,0, z_{0}\right)$, 
- $k_{x}^{i}=i \delta k_{x}$ and $k_{y}^{j}=j \delta k_{y}$ are the discrete wavenumbers about $k_{x}$ and $k_{y}$, respectively,

- $N_{k_{x}}=\frac{\overline{k_{x}}}{\delta k_{x}}$ and $N_{k_{y}}=\frac{\overline{k_{y}}}{\delta k_{y}}$

Adapted criteria should be used for defining the cutoff wavenumbers and the wavenumber resolutions in order to avoid information loss in the numerical integration. These criteria can be established on the basis of previous works [2]. In this paper, criteria were defined for estimating the plate response in the wavenumber space. They can be adapted for the present case. For the wavenumber resolutions, as the wall pressure spectrum generally presents slow variations about the wavenumbers compared to the plate response in the wavenumber space, the same criterion can be used as that defined in Ref. [2]:

$$
\begin{aligned}
& \delta k_{x}=\frac{1}{K} \frac{\eta k_{f}^{\text {plate }}}{2} \\
& \delta k_{y}=\frac{1}{K} \min \left(\frac{\eta k_{f}^{\text {plate }}}{2}, \frac{\eta_{p} k_{f}^{\text {stiffener }}}{2}, \eta_{p} k_{t}^{\text {stiffener }}\right)
\end{aligned}
$$

where:

- $k_{f}^{\text {plate }}$ is the flexural wavenumber of the flat plate,

- $k_{f}^{\text {stiffener }}$ is the flexural wavenumber of the beam-like stiffener,

- $k_{t}^{\text {plate }}$ is the torsional wavenumber of the beam-like stiffener and,

- $K$ is margin coefficient which should be chosen greater than 2 ;

For the cutoff wavenumbers, the criterion defined in Ref. [2] ensures that the regions in the wavenumber space where the plate responses $\tilde{W}\left(k_{x}, k_{y}\right)$ are potentially significant are taken into account. In theory, this criterion should be modified to include the effect of the convective peak of the wall pressure spectrum. For frequencies well above the hydrodynamic coincidence frequency, the convective wavenumber $k_{c}$ is far higher than the natural wavenumber of the plate. Therefore including $k_{c}$ in the cutoff wavenumber criterion will greatly increase the calculation cost, although several studies [27-30] have already shown that for these frequencies the convective peak effect is negligible due to the filtering effect 
of the plate in the wavenumber space. Thus, in practice, it does not seem necessary to include $k_{c}$ in the criterion. To verify this assumption for the numerical application in the next section, we define two criteria, one with and one without the convective wavenumber:

$$
\begin{aligned}
& \text { Criterion 1: } \hat{k_{x}}=\kappa_{x} \max \left(k_{0}, k_{f}^{\text {plate }}\right) \\
& \text { and } \overline{k_{y}}=\kappa_{y} \max \left(k_{0}, k_{f}^{\text {plate }}, k_{f}^{\text {stiffener }}, k_{t}^{\text {stiffener }}\right) \\
& \text { Criterion 2: } \hat{k_{x}}=\kappa_{x} k_{c} \\
& \text { and } \overline{k_{y}}=\kappa_{y} \max \left(k_{0}, k_{f}^{\text {plate }}, k_{f}^{\text {stiffener }}, k_{t}^{\text {stiffener }}\right)
\end{aligned}
$$

with $k_{c}=\omega / U_{c}$ where $U_{c}$ is the convective velocity of the fluid. In these formulas, $\kappa_{x}$ and $\kappa_{y}$ are margin coefficients bigger than one. Different values of these coefficients are tested in the numerical application.

The criteria defined by Eq. (27)-(32) allow us to define the windowing and sampling of the wavenumber spaces from the characteristics of the stiffened plate. Eq. (25) and (26) can then be used to estimate the response of the plate excited by TBL numerically. In the following, the response of the plate can be evaluated in terms of the spectral power density of acceleration $S_{\gamma \gamma}$ which is related to the spectral power density of displacement $S_{w w}$ by relation $S_{\gamma \gamma}=\omega^{4} S_{w w}$.

\section{ANALYSIS OF RESULTS FOR A TEST CASE}

A numerical application is proposed in this section to illustrate several features and results of the present approach. A naval-like structure is considered for this application. The plate is loaded with water and periodically stiffened by beam-like stiffeners having a $\mathrm{T}$ cross-section. The plate and the stiffeners are made of steel. The thickness of the plate is $50 \mathrm{~mm}$. The geometrical and mechanical characteristics of the test case are summarised in Table I. Three values of the stiffener spacing will be considered for the numerical simulation: $\mathrm{d}=1 \mathrm{~m}, \mathrm{~d}=1.35 \mathrm{~m}$ and $\mathrm{d}=1.5 \mathrm{~m}$.

We consider a flow speed of $12 \mathrm{~m} / \mathrm{s}\left(U_{\infty}=12 \mathrm{~m} / \mathrm{s}\right)$. A homogeneous TBL is assumed to be induced on the plate, thus the parameters of this TBL are spatially constant. They are 
TABLE I. Material and geometrical parameters of general use.

\begin{tabular}{lll}
\hline \hline & Plate & Stiffeners \\
\hline$\rho$ & $7800 \mathrm{~kg} / \mathrm{m}^{3}$ & $7800 \mathrm{~kg} / \mathrm{m}^{3}$ \\
$\eta$ & 0.02 & 0.02 \\
$\mathrm{E}$ & $2,1.10^{11}(1+j \eta) \mathrm{Pa}$ & $2,1.10^{11}(1+j \eta) \mathrm{Pa}$ \\
$\nu$ & 0.3 & 0.3 \\
$\mathrm{~h}$ & $50 \mathrm{~mm}$ & - \\
$\mathrm{T}$ cross-section & - & $0.15 \mathrm{mx} 0.08 \mathrm{~m}$ \\
\hline \hline
\end{tabular}

assumed to be $0.047 \mathrm{~m}$ for the thickness and $9.6 \mathrm{~m}$ for the convection velocity $\left(U_{c}=0.8 U_{\infty}\right)$. The interspectrum of the wall pressure due to the turbulent boundary layer is modelled using the first version of the Chase 1987 model (Eq. (39) in Ref. [21]) with the dimensionless parameters given in Ref. [31]: $C_{m}=0.1554, C_{t}=0.0047, b=0.75$. We emphasise that other wall pressure models expressed in the wavenumber space such as those of Corcos [20], Smol'yakov and Tkachenko [26] or Hwang and Geib [32] could be used in the present approach. The Chase 1987 model is used here as an example. An attention has been paid to the definition of the frequency spectrum of the wall pressure. Some models like Corcos' or Smol'yakov's consider single sided spectrum in frequency (i.e. without negative frequencies) where some others like Chase's take a double sided spectrum into account. Furthermore, the angular frequency spectrum can be converted to a frequency dependent spectrum by applying a factor $2 \pi$ (see Ref. [33]). In this paper, a factor $4 \pi$ has been applied to the original Chase spectrum for converting it to a single sided spectrum and showing the results in function of (positive) frequency.

The frequency band of interest is $[100 \mathrm{~Hz}, 1 \mathrm{kHz}]$. For the present case, the hydrodynamic coincidence frequency is lower than $1 \mathrm{~Hz}$ and the critical frequency of the $50 \mathrm{~mm}$ thick plate is around $4.5 \mathrm{kHz}$. Thus the frequencies of investigation are much higher than the hydrodynamic coincidence frequency and lower than the critical frequency. In terms of 
wavenumbers, in the band [100 $\mathrm{Hz}, 1 \mathrm{kHz}]$, we have

$$
k_{0}<k_{f}^{\text {plate }} \ll k_{c}
$$

\section{A. Cutoff wavenumber criterion}

Two possible criteria for the cutoff wavenumber about $k_{x}$ were proposed in section IV: the first, which is more restrictive and less time consuming, is based on the natural wavenumbers of the plate whereas the second is based on the convective wavenumber, $k_{c}$, which allows taking into account the effect of the convective peak. Different studies considering flat plates [27-30] have already shown that the convective peak effect is negligible due to the filtering effect of the plate in the wavenumber space. Thus the first criterion should be reasonable. To verify this assertion for the present stiffened plate, in Table II we propose different results obtained with Eq. (25) and (26), considering the two criteria. Different values of margin coefficients $\kappa_{x}, \kappa_{y}$ are also considered and the comparisons are proposed for two frequencies for which different radiated behaviours of the plate can be observed in a later section. These comparisons show that the first criterion gives the same results as the second if the margin coefficients chosen are not too close to unity. $\kappa_{x}=2, \kappa_{y}=1.5$ seem optimal values for ensuring good convergence with reasonable computing times (i.e. a few seconds). The second criterion is therefore not necessary, confirming that the effect of the convective peak is negligible for the present case. In the rest of the paper, the first criterion with $\kappa_{x}=2, \kappa_{y}=1.5$ is considered.

\section{B. TBL wall pressure and plate displacement for point excitation}

To illustrate the two terms on the right-hand side of Eq. (25) (or Eq. (26)), figure 3 shows the wavenumber spectra of the TBL wall pressure and the stiffened plate displacement (obtained with Eq. (21)). As the spectra are even functions about $k_{y}$, only the part corresponding to the positive $k_{y}$ are plotted. 
TABLE II. Values of $S_{\gamma \gamma}$ at $0 \mathrm{~m}\left(\mathrm{~dB}\right.$, ref. $\left.1 \mu \mathrm{m} . \mathrm{s}^{-2} \cdot H z^{-0.5}\right)$ and $S_{p p}$ at $100 \mathrm{~m}(\mathrm{~dB}$, ref. $\left.1 \mu P a . H z^{-0.5}\right)$ obtained with different truncation criteria.

\begin{tabular}{lllll}
\hline \hline & $S_{\gamma \gamma}(400 \mathrm{~Hz})$ & $S_{\gamma \gamma}(600 \mathrm{~Hz})$ & $S_{p p}(400 \mathrm{~Hz})$ & $S_{p p}(600 \mathrm{~Hz})$ \\
Crit. $1, \kappa_{x}=1.2, \kappa_{y}=1.1$ & $20.7 \mathrm{~dB}$ & $17.0 \mathrm{~dB}$ & $87.9 \mathrm{~dB}$ & $74.9 \mathrm{~dB}$ \\
Crit. $1, \kappa_{x}=2, \kappa_{y}=1.5$ & $20.8 \mathrm{~dB}$ & $17.2 \mathrm{~dB}$ & $88.2 \mathrm{~dB}$ & $76.0 \mathrm{~dB}$ \\
Crit. $1, \kappa_{x}=4, \kappa_{y}=3$ & $20.9 \mathrm{~dB}$ & $17.3 \mathrm{~dB}$ & $88.3 \mathrm{~dB}$ & $76.1 \mathrm{~dB}$ \\
Crit. $2, \kappa_{x}=1.5, \kappa_{y}=1.5$ & $20.9 \mathrm{~dB}$ & $17.3 \mathrm{~dB}$ & $88.3 \mathrm{~dB}$ & $76.1 \mathrm{~dB}$ \\
\hline \hline
\end{tabular}

\section{TBL wall pressure}

The 1987 Chase model is considered here. Thus the TBL wall pressure shown in figure 3 (a) vanishing at a zero wavenumber (in accordance with the Kraichnan-Philips theorem). Moreover, as the wavenumbers considered in this figure are well below the convection wavenumber $\left(k_{c}=\omega / U_{c} \approx 260 \mathrm{~m}^{-1}\right)$, the spectrum for these low-wavenumbers is proportional to the square of the wavenumber [32]. Outside the zero wavenumber region, the pressure spectrum expressed in $\mathrm{dB}$ varies relatively slowly compared to the displacement response of the plate (see figure 3 (b)). The wall pressure for these wavenumbers could be approximated by a constant value without dramatically changing the results of the sum in Eq. (25). The results of this equation would be influenced by the variation of the plate displacement in the wavenumber space.

\section{Plate displacement for point excitation}

On the basis of the $(\mathrm{k}, \mathrm{M})$ reciprocity principle developed in section II.B, we recall that the term $\tilde{W}_{F\left(x_{0}\right)}\left(k_{x}^{i}, k_{y}^{j}, \omega\right)$ in Eq. (25) corresponds to the space Fourier transform of the plate displacement for a harmonic point force excitation at point $\left(x_{0}, 0\right)$ and angular frequency $\omega$. Different features can be observed on the plate displacement given in figure 3 (b):

- the acoustic circle at the bottom-center. It is well-known that only the waves inside 
this acoustic circle will radiate in the far field when the plate is excited by a point force;

- the regions of high displacement amplitudes (in white) which indicate the most excited waves. They will have the largest contribution in the sum of Eq. (25). These regions are approximately in the neighbourhood or inside a circle whose radius is the flexural natural wavenumber of the plate (around $6 \mathrm{~m}^{-1}$ at this frequency). They correspond to propagating Bloch-Floquet waves that are the results of the interaction between the flexural waves of the plate and the flexural/torsional waves of the stiffeners spaced periodically. This type of wave plays a strong role in the dynamic behaviour of periodically stiffened structures, making it necessary to focus on this point.

\section{Propagating Bloch-Floquet waves}

As proposed in Ref. [1], the wavenumbers corresponding to these propagating BlochFloquet waves can be predicted by finding the roots $\left(k_{x}^{\mathrm{BF}}, k_{y}^{\mathrm{BF}}\right)$ of the denominator of Eq. (21) (assuming the system as conservative):

$$
\begin{aligned}
\Delta\left(k_{x}^{\mathrm{BF}}, k_{y}^{\mathrm{BF}}\right)=\left[1+S_{0}\left(k_{x}^{\mathrm{BF}}, k_{y}^{\mathrm{BF}}\right) Z_{p}\left(k_{y}^{\mathrm{BF}}\right)\right]\left[1+S_{2}\left(k_{x}^{\mathrm{BF}}, k_{y}^{\mathrm{BF}}\right) A_{p}\left(k_{y}^{\mathrm{BF}}\right)\right] & \\
& -S_{1}\left(k_{x}^{\mathrm{BF}}, k_{y}^{\mathrm{BF}}\right)^{2} Z_{p}\left(k_{y}^{\mathrm{BF}} A_{p}\left(k_{y}^{\mathrm{BF}}\right)=0\right.
\end{aligned}
$$

This is the condition for the existence of free waves in the periodically stiffened plate. If $\left(k_{x}^{\mathrm{BF}}, k_{y}^{\mathrm{BF}}\right)$ are real, the free waves propagate without attenuation across the plate. They are called propagating Bloch-Floquet waves. When the forced response of the plate is considered, these propagating Bloch-Floquet waves dominate in the response of the plate and the spectrum in the wavenumber space exhibits the highest amplitudes for these Bloch-Floquet wavenumbers. In other terms, as $\left(k_{x}^{\mathrm{BF}}, k_{y}^{\mathrm{BF}}\right)$ cancels the denominator of Eq. (21), assuming

the system as conservative, if we introduce $k_{x}=k_{x}^{\mathrm{BF}}$ and $k_{y}=k_{y}^{\mathrm{BF}}$ in Eq. (21), considering the system is non conservative (i.e. considering the damping effect), the displacement amplitude will be higher than the amplitude obtained for $k_{x} \neq k_{x}^{\mathrm{BF}}$ and $k_{y} \neq k_{y}^{\mathrm{BF}}$. 
As indicated in previous studies [1,34], explicit expressions for $k_{x}^{\mathrm{BF}}$ and $k_{y}^{\mathrm{BF}}$ cannot be derived when the plate is loaded with a fluid. In order to approximate these Bloch-Floquet wavenumbers, we define an algorithm to extract the real values of $\left(k_{x}^{\mathrm{BF}}, k_{y}^{\mathrm{BF}}\right)$ such that $\Delta\left(k_{x}^{\mathrm{BF}}, k_{y}^{\mathrm{BF}}\right)$ is lower than a predefined low value $\epsilon$. Two properties of $\Delta\left(k_{x}^{\mathrm{BF}}, k_{y}^{\mathrm{BF}}\right)$ allow us to restrict the interval to find $k_{x}^{\mathrm{BF}}$ :

- $S_{0}, S_{1}, S_{2}$ are $\frac{2 \pi}{d}$-periodic function of $k_{x}$, thus

$$
\left.\left.k_{x}^{\mathrm{BF}}=\bar{k}_{x}^{\mathrm{BF}}+\frac{2 \pi n}{d}, \forall n \in Z \quad \text { with } \quad \bar{k}_{x}^{\mathrm{BF}} \in\right]-\frac{\pi}{d}, \frac{\pi}{d}\right]
$$

$\bar{k}_{x}^{\mathrm{BF}}$ can be called the propagating Bloch-Floquet wavenumbers of the first Brillouin zone. An infinite number of Bloch-Floquet wavenumbers $k_{x}^{\text {BF }}$ (i.e. roots) can be deduced from $\bar{k}_{x}^{\mathrm{BF}}$.

- $\Delta\left(k_{x}^{\mathrm{BF}}, k_{y}^{\mathrm{BF}}\right)$ is an even function about $k_{x}$ thus we can restrict the interval $\left[0, \frac{\pi}{d}\right]$ to find the $\operatorname{root} \bar{k}_{x}^{\mathrm{BF}}$.

The algorithm is then written as follows:

$$
\text { For } k_{y}^{i} \in\left[0, \bar{k}_{y}\right], \Delta_{\min }\left(k_{y}^{i}\right)=\min _{k_{c} \in\left[0, \frac{\pi}{d}[\right.} \Delta\left(k_{x}, k_{y}^{i}\right)
$$

- if $\Delta_{\min }\left(k_{y}^{i}\right)<\epsilon$ then $k_{y}^{i}$ is a propagating BF wave characterised by

$$
k_{y}^{\mathrm{BF}}=k_{y}^{i} \quad \text { and } \quad \bar{k}_{x}^{\mathrm{BF}} \in\left[0, \frac{\pi}{d}\right] / \Delta\left(\bar{k}_{x}^{\mathrm{BF}}, k_{y}^{\mathrm{BF}}\right)=\Delta\left(k_{y}^{\mathrm{BF}}\right)
$$

- if $\Delta_{\min }\left(k_{y}^{i}\right) \geq \epsilon$ then $k_{y}^{i}$ is an evanescent wave.

The BF wavenumbers presented in this paper were obtained with $\epsilon=0.05$ in the previous algorithm.

The values of $k_{y}^{\mathrm{BF}}$ obtained are indicated in figure 3 (b) by a white circle on the $k_{y}$ axis. These values correspond well to the $k_{y}$ values for which the displacement spectrum exhibits the highest amplitudes. Two sets of values can be observed in this figure $\left(k_{y} \in[0,3.3] \mathrm{rad} / \mathrm{m}\right.$ and $\left.k_{y} \in[4.9,5.1] \mathrm{rad} / \mathrm{m}\right)$. A set of $k_{y}^{\mathrm{BF}}$ is called "pass band" and corresponds to a set of waves propagating along the plate. A set of $k_{y}$ between two pass bands is called a "stop 
band" and corresponds to a set of evanescent waves (i.e. waves with strong attenuation across the plate). For the case of figure 3 (b), two pass bands and one stop band can be seen. These bands are characteristic of the displacement response of the stiffened plate excited by a point source. The numbers and the positions of these bands depend on the frequency considered, as illustrated in figure 4 . The values of $k_{y}^{\mathrm{BF}}$ were plotted for different frequencies between $100 \mathrm{~Hz}$ and $1000 \mathrm{~Hz}$. It can be seen that this graph is similar in form to the graphs obtained by different authors studying stiffened structures $[1,35]$. In-depth analyses of these types of graph can be found in these references, but they are not given here for the sake of brevity in the present paper. Figure 4 shows that the number of pass band and their $k_{y}$ positions increases with frequency. It can be seen that some pass bands are contiguous. This is the case, for instance, at $400 \mathrm{~Hz}$, where the first pass band observed in figure $3(\mathrm{~b})\left(k_{y} \in[0,3.5] \mathrm{rad} / \mathrm{m}\right)$ is subdivided into two pass bands $k_{y} \in[0,2.1] \mathrm{rad} / \mathrm{m}$ and $k_{y} \in[2.2,3.3] \mathrm{rad} / \mathrm{m}$.

As we shall see in the next section, these pass bands and their positions in the wavenumber space have a strong influence on the pressure radiated by the plate excited by a TBL.

\section{Vibro-acoustic response of the plate excited by a TBL}

The vibration of and the sound radiated from the stiffened plate excited by a TBL $(\mathrm{d}=1 \mathrm{~m})$ is estimated with Eq. (25) and Eq. (26) in the frequency band [100-1000 Hz]. The computations are performed on a standard PC using MATLAB and take only a few seconds for one frequency.

The results are given in figure 5 for two observation points on the plate: the first, on a rib (i.e. $\mathrm{x}=0 \mathrm{~m}$ ); the second between two ribs (i.e. $\mathrm{x}=0.3 \mathrm{~m}$ ). The results for a $50 \mathrm{~mm}$ thick plate (without ribs) are proposed for comparison. As can be expected, the response point located on a stiffener shows acceleration spectra lower than those of the point located between two stiffeners. On the other hand, it can be seen that the vibratory response of the stiffened plate is relatively smooth according to frequencies. This indicates that the 
pass bands do not have a significant effect on the vibratory response of the plate for a TBL excitation. The $k_{y}$ positions and lengths of the pass bands depend on frequency, but as the plate response due to the TBL is obtained by summing all the $k_{y}$, the acceleration spectrum does not depend significantly on these positions and lengths.

In contrast, as can be observed in figure 6, the pressure spectra radiated at different distances of the plate may vary significantly as a function of frequency: for the point located $10 \mathrm{~m}$ from the plate, the radiated pressure can be $15 \mathrm{~dB}$ higher in certain frequency bands than in others. This behaviour becomes more prevalent as the distance from the plate increases. However, a limit exists beyond which the pressure spectra remain unchanged. To analyse this phenomenon, we propose in figure 7 summing the terms of Eq. (26), $\left|\tilde{W}_{M\left(z_{0}\right)}\left(k_{x}^{i}, k_{y}^{j}, \omega\right)\right|^{2} \phi_{p p}\left(k_{x}^{i}, k_{y}^{j}, \omega\right)$. We recall that the wall pressure spectra $\phi_{p p}\left(k_{x}^{i}, k_{y}^{j}, \omega\right)$ are roughly constant for these wavenumbers. The variations of the summing terms are therefore due to the displacement spectrum for a monopole source located at $z_{0}$ from the plate, $\tilde{W}_{M\left(z_{0}\right)}\left(k_{x}^{i}, k_{y}^{j}, \omega\right)$. Figure 7 shows that a filtering effect of the fluid medium occurs when the distance from the plate increases. Only wavenumbers inside the acoustic circle (i.e. $\sqrt{k_{x}^{2}+k_{y}^{2}} \leq k_{0}$ ) or inside periodic copies of the acoustic circle have significant contributions when the observation point is far from the plate. The periodicity of these copies is $\frac{2 \pi}{d}$. For a plate without stiffeners, the periodic copies are not observed. Only wavenumbers inside the acoustic circle gave a contribution corresponding to the waves propagating into the fluid medium. This explains the lower levels in figure 6 for the plate alone compared to the stiffened plate.

The periodic copies are clearly the consequence of the periodic stiffeners of the plate. They can be interpreted as the acoustic wave as seen from the stiffeners. Mathematically, it corresponds to a discrete Fourier transform of the spatial field with a resolution corresponding to the stiffener spacing. The result of this transform is a periodic spectrum with $\frac{2 \pi}{d}$ as period.

As shown in figure 7, this filtering effect increases with distance from the plate. This explains the previous observation in figure 6 concerning the effect of distance. A threshold 
effect is reached since the contributions of all the wavenumbers outside the acoustic circle and its copies can be neglected.

The difference of frequency dependence observed between the plate vibration and the radiated pressure (see figures 5 and 6) can now be explained by figure 8 in which the summing terms of Eq. (25) and (26) are plotted for two frequencies: $400 \mathrm{~Hz}$ and $600 \mathrm{~Hz}$. These frequencies were chosen due to the considerable differences in the plate's radiation efficiency $\left(88.2 \mathrm{~dB}\right.$ at $400 \mathrm{~Hz}$ against $76 \mathrm{~dB}$ at $600 \mathrm{~Hz}$ for $z_{0}=10 \mathrm{~m}$ ). The pass bands about $k_{y}$ are indicated in figure 8 (by white circles on the $k_{y}$ axis) The positions of these bands change with frequency, but the sum of terms shown in figures 8 (a) and 8 (c) give similar results. The vibratory response between the two frequencies is therefore similar. On the contrary, for the radiated pressure, the relative positions of the pass band compared to the acoustic domain $\left|k_{y}\right|<k_{0}$ play an important role. Indeed, at $400 \mathrm{~Hz}$, a pass band is present in the acoustic domain. The propagating Bloch-Floquet waves fall within the acoustic circle or its periodic copies. They radiate in the far field and lead to the relatively high radiated noise level that can be observed. At $600 \mathrm{~Hz}$, only a stop band occurs in the acoustic wavenumber. The propagating Bloch-Floquet waves cannot radiate in the far field, leading to a relatively low radiated noise level. This explains the difference of radiated noise between the two frequencies.

By studying the change of the pass band positions with frequency, it is possible to predict if the frequency spectrum of the radiated pressure in the far field shows a bump or a hollow. To do this, the graph of figure 4 is not completely appropriate as it does not permit indicating if the propagating Bloch-Floquet waves occur in the acoustic circle or its copies (but only if $\left|k_{y}^{\mathrm{BF}}\right| \leq k_{0}$ ).

As the $k_{y}^{\mathrm{BF}}$ Bloch-Floquet wavenumbers (see Eq. (35)) and the periodic copies of the acoustic circle are $\frac{2 \pi}{d}$-periodic about $k_{x}$, it is only necessary to verify if the Bloch-Floquet wavenumber of the first Brillouin zone is contained inside the acoustic circle. In this case, the Bloch-Floquet wavenumber considered should be $\bar{k}^{\mathrm{BF}}=\sqrt{\left(\bar{k}_{x}^{\mathrm{BF}}\right)^{2}+\left(k_{y}^{\mathrm{BF}}\right)^{2}}$. If $\bar{k}^{\mathrm{BF}} \leq k_{0}$, a propagating Bloch-Floquet wave radiates in the far field. It should be emphasised that 
this criterion does not indicate the magnitude of the BF wave or if it is located in the first Brillouin zone or in another zone. It only indicates whether a propagating Bloch-Floquet wave radiates in the far field or not.

To verify this criterion, we consider three plates with different stiffener spacings: $1 \mathrm{~m}$, $1.35 \mathrm{~m}$ and $1.5 \mathrm{~m}$. Their Bloch-Floquet wavenumbers $\bar{k}^{\mathrm{BF}}$ are plotted versus frequency in figure 9 and their values are compared to the acoustic wavenumber, $k_{0}$. A set of frequencies for which almost one BF wave radiates are called "frequency pass bands". These bands are symbolised by black circles on the frequency axis of figure 9. When the stiffener spacing increases, it can be seen that their number increases and their positions shift toward the low frequencies. This must be reflected in the spectra of the radiated pressure when the plate is excited by a TBL. This is shown in figure 10 which superposes the spectrum of the radiated pressure level at $10 \mathrm{~m}$ from the plate over the frequency pass bands determined in the previous figure. The bumps of radiated noise clearly occur inside the frequency pass bands. This validates our assumption about the radiation efficiency of the BF waves. It is noteworthy that the length of the bumps is not always equal to the length of the frequency pass bands. This may be explained by the fact that these bands indicate only that a propagating BF wave radiates, without indicating its magnitude.

In conclusion, this section permitted illustrating typical results obtained by the present approach and highlighting the effect of the pass band on the radiated noise when the stiffened plate is excited by a TBL.

\section{CONCLUSIONS}

A process based on the so-called $(\mathrm{k}, \mathrm{M})$ reciprocity relation was proposed in this paper to estimate the vibro-acoustic response of a periodically stiffened plate under a turbulent layer excitation. The $(\mathrm{k}, \mathrm{M})$ reciprocity principle allows avoiding time-consuming calculations of plate responses excited by different plane waves representing TBL excitation. Indeed, only the displacement field of the panel in the wavenumber domain for a point excitation is 
required with the methodology proposed. In accordance with this principle, the excitation point must be located at the point for which the response of the plate excited by the TBL is to be estimated. To predict the flow induced noise from the stiffened plate at a receiving point, the displacements in the wavenumber space for a monopole source located at the receiving point must be calculated. For the case of an infinite periodically stiffened plate, analytical expressions of the displacement fields are available for a mechanical point force on the plate or for an acoustic monopole source in the fluid. The numerical calculations with the present approach are then reduced to a numerical integration for which criteria have been proposed. Analysis of results from a naval test case highlights a difference in the effect of stiffeners between the vibration of the plate and the far field pressure radiated for TBL excitation: although adding stiffeners to a flat plate slightly reduces its vibrations, it enhances the noise in the far field in certain frequency bands. It was shown that this enhancement occurs when propagating Bloch-Floquet waves radiate in the far field, that is to say when their wavenumbers (in the first Brillouin zone) are lower than the acoustic wavenumber. Thus the frequency bands for which the stiffened plate may radiate more noise than the flat plate can be predicted by estimating the wavenumbers of the propagating Bloch-Floquet waves.

The present methodology was applied to infinite plates with regularly spaced stiffeners and can be extended to non-periodically stiffened plates of finite size. The theoretical formulation of section 2 and the $(\mathrm{k}, \mathrm{M})$ reciprocity relation remain valid. For this case, the displacement field in the wavenumber space could be estimated from Discrete Fourier Transforms of the displacement field in physical space for a point excitation. From the modelling standpoint, this field can be estimated using classical discretization approaches like finite element and boundary element methods. From the experimental standpoint, a scanning laser vibrometer can be used to measure the vibrations of the plate excited by a shaker or a loudspeaker. These two possibilities should be studied in the future. They are necessary to validate the present process experimentally. The results of the present approach concerning panels of finite sizes will permit comparisons with measurements in a wind tunnel or hydrodynamic tunnel. Moreover, it would be possible to verify whether the stiffener effect of 
radiated noise enhancement is always observed on finite panels. For submarine applications, which is one of the authors' areas of interest, this process could be used for estimating the vibration and radiated noise of the pressure hull excited by a turbulent flow. As the characterization of the wall pressure fluctuations proposed in the literature generally concerns canonical flat plates, it is logical to assume that the wall pressure models established for plates give a satisfactory approximation for cylindrical shells. The vibro-acoustic behaviour of a submerged irregularly ribbed shell can be predicted by the Circumferential Admittance Approach [36]. This approach is based on coupling an analytical model of the shell submerged in a fluid with finite element models of its internal frames (i.e. stiffeners, bulkheads). One intermediate result of this approach is the radial displacement field of the shell in the wavenumber space. This result can be used directly in the present approach for predicting the response of the non-periodically stiffened shell excited by the TBL.

\section{APPENDIX A: RESPONSE OF THE PERIODICALLY STIFFENED PLATE IN THE WAVENUMBER SPACE}

The problem described in section III can be solved in the wavenumber domain by using the space-Fourier transform defined by

$$
f(x, y) \rightarrow \tilde{f}\left(k_{x}, k_{y}\right)=\int_{R^{2}} f(x, y) e^{-j\left(k_{x} x+k_{y} y\right)} \mathrm{d} x \mathrm{~d} y
$$

where $k_{x}$ and $k_{y}$ are the wavenumbers for the $x$ and $y$ axis.

By applying this transform to the equation of motion (16), we deduce

$$
\begin{aligned}
& {\left[D^{*}\left(k_{x}^{2}+k_{y}^{2}\right)^{2}-\omega^{2} \rho h\right] \tilde{W}\left(k_{x}, k_{y}\right)=\tilde{F}-\tilde{p}\left(k_{x}, k_{y}, 0\right)} \\
& -\frac{1}{d}\left(E_{p}^{*} I_{x} k_{y}^{4}-\omega^{2} \rho_{p} S_{p}\right) \sum_{n \in \mathbb{Z}} \tilde{W}\left(k_{x}+\frac{2 \pi n}{d}, k_{y}\right) \\
& \quad-\frac{k_{x}}{d}\left(G_{p}^{*} J_{p} k_{y}^{2}-\omega^{2} \rho_{p} I_{0}\right) \sum_{n \in \mathbb{Z}}\left(k_{x}+\frac{2 \pi n}{d}\right) \tilde{W}\left(k_{x}+\frac{2 \pi n}{d}, k_{y}\right)
\end{aligned}
$$

where $\tilde{W}\left(k_{x}, k_{y}\right)$ and $\tilde{p}\left(k_{x}, k_{y}, 0\right)$ are the plate displacement and the wall pressure in the wavenumber space, respectively. 
On the other hand, by taking the space-Fourier transform of the Helmholtz and Euler equation and resolving the $z$ differential equation and applying the Sommerfeld conditions on $\Sigma_{\infty}$, the pressure may be written as

$$
\begin{cases}\tilde{p}\left(k_{x}, k_{y}, z\right)=\frac{\rho_{0} \omega^{2} e^{k_{z} z}}{k_{z}} \tilde{W}\left(k_{x}, k_{y}\right) \quad \text { if } \sqrt{k_{x}^{2}+k_{y}^{2}} \neq k_{0}, \\ \tilde{p}\left(k_{x}, k_{y}, z\right)=C, \tilde{W}\left(k_{x}, k_{y}\right)=0 \quad \text { if } \sqrt{k_{x}^{2}+k_{y}^{2}}=k_{0},\end{cases}
$$

where $C$ is a constant and $k_{z}=-\sqrt{k_{x}^{2}+k_{y}^{2}-k_{0}^{2}}$ with the convention $\operatorname{Im}\left(k_{y}\right) \leq 0$ if $\operatorname{Re}\left(k_{y}\right)=$ 0.

Introducing Eq. (A3) in Eq. (A2), one has if $\sqrt{k_{x}^{2}+k_{y}^{2}} \neq k_{0}$

$$
\tilde{W}\left(k_{x}, k_{y}\right)=\frac{1}{Z\left(k_{x}, k_{y}\right)}\left[\tilde{F}-Z_{p}\left(k_{y}\right) \xi\left(k_{x}, k_{y}\right)-k_{x} A_{p}\left(k_{y}\right) \zeta\left(k_{x}, k_{y}\right)\right]
$$

where

$$
\begin{aligned}
Z\left(k_{x}, k_{y}\right) & =D^{*}\left(k_{x}^{2}+k_{y}^{2}\right)^{2}-\rho \omega^{2} h-\frac{\rho_{0} \omega^{2}}{\sqrt{k_{x}^{2}+k_{y}^{2}-k_{0}^{2}}}, \\
Z_{p}\left(k_{y}\right) & =\frac{1}{d}\left(E_{p}^{*} I_{x} k_{y}^{4}-\omega^{2} \rho_{p} S_{p}\right), \\
\text { and } A_{p}\left(k_{y}\right) & =\frac{1}{d}\left(G_{p}^{*} I_{p} k_{y}^{2}-\omega^{2} \rho_{p} I_{0}\right)
\end{aligned}
$$

are respectively the fluid-loaded plate impedance, the flexural and the torsional stiffener impedances, and

$$
\left\{\begin{array}{l}
\xi\left(k_{x}, k_{y}\right)=\sum_{n \in \mathbb{Z}} \tilde{W}\left(k_{x}+\frac{2 \pi n}{d}, k_{y}\right) \\
\zeta\left(k_{x}, k_{y}\right)=\sum_{n \in \mathbb{Z}}\left(k_{x}+\frac{2 \pi n}{d}\right) \tilde{W}\left(k_{x}+\frac{2 \pi n}{d}, k_{y}\right)
\end{array}\right.
$$

are a notation for convenience.

Note that $\xi$ and $\zeta$ are $\frac{2 \pi}{d}$-periodic with respect to $k_{x}$ (i.e. $\left.\xi\left(k_{x}+\frac{2 \pi n}{d}, k_{y}\right)=\xi\left(k_{x}, k_{y}\right)\right)$. In order to evaluate these quantities, $\tilde{W}$ is replaced by its periodised expression in the sums 
of Eq. (A8)

$$
\left\{\begin{aligned}
\xi\left(k_{x}, k_{y}\right)= & \sum_{n \in \mathbb{Z}} \frac{1}{Z\left(k_{x}+\frac{2 \pi n}{d}, k_{y}\right)}\left[\tilde{F}-Z_{p}\left(k_{y}\right) \xi\left(k_{x}, k_{y}\right)\right. \\
& \left.-\left(k_{x}+\frac{2 \pi n}{d}\right) A_{p}\left(k_{y}\right) \zeta\left(k_{x}, k_{y}\right)\right] \\
\zeta\left(k_{x}, k_{y}\right)= & \sum_{n \in \mathbb{Z}} \frac{\left(k_{x}+\frac{2 \pi n}{d}\right)}{Z\left(k_{x}+\frac{2 \pi n}{d}, k_{y}\right)}\left[\tilde{F}-Z_{p}\left(k_{y}\right) \xi\left(k_{x}, k_{y}\right)\right. \\
& \left.-\left(k_{x}+\frac{2 \pi n}{d}\right) A_{p}\left(k_{y}\right) \zeta\left(k_{x}, k_{y}\right)\right] .
\end{aligned}\right.
$$

Now, the excitation $\tilde{F}$ is expressed in the form: $\tilde{F}=P_{0} \Lambda, P_{0}$ being the amplitude of the excitation source and $\Lambda$ being given by

$$
\Lambda\left(k_{x}, k_{y}\right)= \begin{cases}e^{-j k_{x} x_{0}} & \text { for a force excitation at }\left(x_{0}, 0\right) \\ \frac{e^{-k_{z} z_{0}}}{-k_{z}} & \text { for a monopole source at }\left(0,0, z_{0}\right) .\end{cases}
$$

Then Eq. (A9) can be rewritten

$$
\left\{\begin{aligned}
\xi\left(k_{x}, k_{y}\right) & =P_{0} T_{0}-S_{0} Z_{p}\left(k_{y}\right) \xi\left(k_{x}, k_{y}\right) \\
& -S_{1} A_{p}\left(k_{y}\right) \zeta\left(k_{x}, k_{y}\right) \\
\zeta\left(k_{y}, k_{y}\right) & =P_{0} T_{1}-S_{1} Z_{p}\left(k_{y}\right) \xi\left(k_{x}, k_{y}\right) \\
& -S_{2} A_{p}\left(k_{y}\right) \zeta\left(k_{x}, k_{y}\right)
\end{aligned}\right.
$$

where

$$
\left\{\begin{array}{r}
S_{p}=\sum_{n \in \mathbb{Z}} \frac{\left(k_{x}+\frac{2 \pi n}{d}\right)^{p}}{Z\left(k_{x}+\frac{2 \pi n}{d}, k_{y}\right)} \\
T_{p}=\sum_{n \in \mathbb{Z}} \frac{\left(k_{x}+\frac{2 \pi n}{d}\right)^{p} \Lambda\left(\frac{2 \pi n}{d}, k_{y}\right)}{Z\left(k_{x}+\frac{2 \pi n}{d}, k_{y}\right)} .
\end{array}\right.
$$

Eq. (A11) is a linear system with unknowns $\xi$ and $\zeta$. Solving it, their expressions are obtained and can be replaced in Eq. (A4). This operation gives the final result:

$$
\begin{aligned}
\tilde{W}\left(k_{x}, k_{y}\right)=\frac{P_{0}}{Z\left(k_{x}, k_{y}\right)}[ & \Lambda\left(k_{x}, k_{y}\right) \\
& -\frac{Z_{p}\left(k_{y}\right)\left(T_{0}\left(1+S_{2} A_{p}\left(k_{y}\right)\right)-T_{1} S_{1} A_{p}\left(k_{y}\right)\right)}{\Delta} \\
& \left.\quad-\frac{k_{x} A_{p}\left(k_{y}\right)\left(T_{0} S_{1} Z_{p}\left(k_{y}\right)-T_{1}\left(1+S_{0} Z_{p}\left(k_{y}\right)\right)\right)}{\Delta}\right] .
\end{aligned}
$$

with

$$
\Delta=\left(\left(1+S_{0} Z_{p}\left(k_{y}\right)\right)\left(1+S_{2} A_{p}\left(K_{y}\right)\right)-S_{1}^{2} Z_{p}\left(k_{y}\right) A_{p}\left(k_{y}\right)\right)
$$




\section{REFERENCES}

1 B.R. MACE. Periodically stiffened fluid-loaded plates, i: Response to convected harmonic pressure and free wave propagation. Journal of Sound and Vibration, 73(4):473-486, 1980.

2 L. MAXIT. Wavenumber space and physical space responses of a periodically ribbed plate to a point drive: A discrete approach. Applied Acoustics, 70:563-578, 2008.

3 W.A. STRAWDERMAN. Turbulence-induced plate vibrations: an evaluation of finiteand infinite-plate models. J. Acoust. Soc. Am., 46(5):1294-1295, 1969.

4 W.A. STRAWDERMAN and R.A. CHRISTMAN. Turbulence-induced plate vibrations: some effects of fluid loading on finite and infinite plates. J. Acoust. Soc. Am., 52(5): $1537-1552,1971$.

${ }^{5}$ H.G. DAVIS. Sound from turbulent boundary layer excited panel. J. Acoust. Soc. Am., 49(3):878-889, 1971.

6 F.A. AUPPERLE and R.F. LAMBERT. Acoustic radiation from plates excited by flow noise. Journal of Sound and Vibration, 26(2):223-245, 1972.

7 M.L. RUMERMAN. Frequency-flow speed dependence of structural response to turbulent boundary layer pressure excitation. J. Acoust. Soc. Am., 91(2):907-910, 1992.

8 W.R. GRAHAM. Boundary layer induced noise in aircraft, part i: the flat panel model. Journal of Sound and Vibration, 192(1):101-120, 1995.

9 S.H. KO and H.H. SCHLOEMER. Calculations of turbulent boundary layer pressure fluctuations transmitted into a viscoelastic layer. J. Acoust. Soc. Am., 85(4):1469-1477, 1989.

10 S.H. KO and H.H. SCHLOEMER. Flow noise reduction techniques for a planar array of hydrophones. J. Acoust. Soc. Am., 92(6):3409-3424, 1992.

11 C. MAURY, P. GARDONIO, and S.J. ELLIOTT. A wavenumber approach to modelling the response of a randomly excited panel, part i: general theory. Journal of Sound and Vibration, 252(1):83-113, 2002.

12 W.A. STRAWDERMAN. Wavector-frequency analysis with applications to acoustics. 
Technical Report 8209, NUSC, 1988.

13 D. MAZZONI. An efficient approximation for the vibro-acoustic response of a turbulent boundary layer excited panel. Journal of Sound and Vibration, 264(4):951-971, 2003.

14 D.J. MEAD. Wave propagation in continuous periodic structures: research contributions from southampton, 1964-1995. Journal of Sound and Vibration, 190(3):495-524, 1996.

15 M.L. RUMERMAN. Vibration and wave propagation in ribbed plates. J. Acoust. Soc. Am., 57(2):370-380, 1974.

16 G. MAIDANIK and J. DICKEY. Reflection of incident pressures waves by ribbed panels. J. Acoust. Soc. Am., 90(4):2124-2134, 1991.

17 M.L. RUMERMAN. Estimation of broadband acoustic power radiated from a turbulent boundary layer-driven reinforced finite plate section due to rib and boundary forces. $J$. Acoust. Soc. Am., 111(3):1274-1284, 2001.

18 M. AUCEJO. Vibro-acoustique des structures immerges sous coulement turbulent. PhD thesis, Institut National des Sciences Appliques de Lyon, 2010.

19 F. FAHY. Some applications of the reciprocity principle in experimental vibroacoustics. Acoustical Physics, 49(3):262-277, 2003.

20 G.M. CORCOS. The resolution of pressure in turbulence. J. Acoust. Soc. Am., 35: 192-199, 1963.

21 D.M. CHASE. The character of the turbulent wall pressure spectrum at subconvective wavenumbers and a suggested comprehensive model. Journal of Sound and Vibration, 112(1):125-147, 1987.

22 G. CHERTOCK. General reciprocity relation. J. Acoust. Soc. Am., 34:989, 1962.

23 T. TEN WOLDE. Reciprocity measurements in acoustical and mechano-acoustical systems. Review of theory and applications. Acta Acustica united with Acustica, 96:1-13, 2010.

24 D. GUEORGUIEV and J.G. MCDANIEL and P. DUPONT and L.B. FELSEN. Analysis of floquet wave generation and propagation in a plate with multiple arrays of line attachments. Journal of Sound and Vibration, 96(5):819-840, 2000. 
25 C. KAUFFMANN. Efficiency of a monopole source in the vicinity of a water-loaded plate. Journal of Sound and Vibration, 221(2):251-272, 1999.

26 A.V. SMOL'YAKOV and V.M. TKACHENKO. Model of a field of pseudosonic turbulent wall pressures and experimental data. Soviet Physics Acoustics, 37(6):627-631, 1991.

27 A.O. BORISYUK and V.T. GRINCHENKO. Vibration and noise generation by elastic elements excited by a turbulent flow. Journal of Sound and Vibration, 204(2):213-237, 1997.

28 W.K. BLAKE. Mechanics of flow-induced sound and vibration. Volume II: Complex Flow-structure interaction. Academic Press, Inc. Orlando, Florida, 1986.

29 W.R. GRAHAM. A comparison of models for the wavenumber-frequency spectrum of turbulent boundary layer pressures. Journal of Sound and Vibration, 204(2):541-565, 1997.

30 L. MAXIT and C. AUDOLY. Hydrodynamic noise prediction inside a sonar dome: estimation of injected power from the wavenumber-frequency spectrum of the turbulent wall pressure. In Proceedings of NOVEM 2005, Saint-Raphael, 2005.

31 H.F. HWANG, W.K. BONNESS, and S.A. HAMBRIC. Comparision of semi-empirical models for turbulent boundary layer wall presssure spectra. Journal of Sound and Vibration, 319:199-217, 2009.

32 Y.F. HWANG and F.E. GEIB. Estimation of the wavevector-frequency spectrum of turbulent boundary later wall pressure by multiple linear regression. Journal of Vibration, Acoustics, Stress and Reliability in Design - Transactions of the ASME, 106:334-344, 1984.

33 S.A HAMBRIC and Y.F. HWANG and W.K. BONNESS. Vibrations of plates with clamped and free edges excited by low-speed turbulent boundary layer flow. Journal of Fluids and Structures, 19:93-110, 2004.

34 D.J. MEAD. Plates with regular stiffening in acoustic media: Vibration and radiation. J. Acoust. Soc. Am., 88:391-401, 1990.

35 C.H. HODGES, J. POWER, and J. WOODHOUSE. The low frequency vibration of a 
ribbed cylinder, part i : Theory. Journal of Sound and Vibration, 10(2):219-235, 1985.

36 L. MAXIT and J.M. GINOUX. Sound radiated by a submerged irregularly ribbed shell: the circumferentual admittance approach. J. Acoust. Soc. Am., 128(1):137-151, 2010. 


\section{LIST OF FIGURES}

FIG. 1: Panel excited by a TBL with flow speed $U_{\infty}$.

FIG. 2: Lyamshev reciprocity relation for elastic structures excited by point forces.

FIG. 3: (a), Wavenumber spectrum of the TBL wall pressure at $400 \mathrm{~Hz}$, Chase model (dB, ref. $\left.1 \mu \mathrm{Pa} \cdot \mathrm{Hz}^{-0.5} \cdot \mathrm{rad}^{-1}\right)$. (b), Wavenumber spectrum of the plate displacement at $400 \mathrm{~Hz}$ for a force excitation on a rib $\left(\mathrm{dB}\right.$, ref. $\left.1 \mathrm{~m} . \mathrm{N}^{-1}\right)$. Stiffener spacing: $1 \mathrm{~m}$. Propagation zones are indicated by white circles on the $k_{y}$ axis.

FIG. 4: Propagation zones (lined) and attenuation zones in $\left(f, k_{y}\right)$ space for the plate with rib spacing of $1 \mathrm{~m}$.

FIG. 5: Frequency spectrum of the plate acceleration for a TBL excitation $\left(S_{\gamma \gamma}=\omega^{4} S_{w w}\right)$. Plate without ribs, solid line; plate with ribs spacing of $1 \mathrm{~m}$, response at $\mathrm{x}=0 \mathrm{~m}$, dashed line; and response at $\mathrm{x}=0.3 \mathrm{~m}$, dashed-dotted line. $\left(\mathrm{dB}\right.$, ref. $\left.1 \mu \mathrm{m} \cdot \mathrm{s}^{-2} \cdot \mathrm{Hz}^{-0.5}\right)$.

FIG. 6: Frequency spectrum of the radiated pressure of the stiffened plate at different positions $\left(0,0, z_{0}\right)$ from the plate: $z_{0}=0.05 \mathrm{~m}$ (solid line); $z_{0}=0.2 \mathrm{~m}$ (dash line); $z_{0}=1$ $\mathrm{m}$ (dashed-dotted line); $z_{0}=10 \mathrm{~m}$ (dotted line); Frequency spectrum of the radiated pressure at $z_{0}=10 \mathrm{~m}$ of the plate without stiffeners, symbolized by point marker $(\mathrm{dB}$, ref. $\left.1 \mu \mathrm{Pa} . \mathrm{Hz}^{-0.5}\right)$.

FIG. 7: Values of the summing terms of Eq. (26) in the wavenumber space for different positions of the monopole source: (a), $z_{0}=0.2 \mathrm{~m}$; (b), $z_{0}=1 \mathrm{~m} ;(\mathrm{c}), z_{0}=10 \mathrm{~m}$. Frequency: $600 \mathrm{~Hz}\left(\mathrm{~dB}\right.$, ref. $1 \mu$ Pa.m.Hz $\left.{ }^{-0.5} \cdot \operatorname{rad}^{-1}\right)$.

FIG. 8: (a), (c), values of the summing terms of Eq. (25) for an observation point on the plate at $(0,0)$. Propagation zones symbolised by white circles on the $k_{y}$ axis; (b), (d), values of the summing terms of Eq. (26) for an observation point in the acoustic domain at (0,0,10 m). Two frequencies: (a), (b), $400 \mathrm{~Hz}$; (c), (d), $600 \mathrm{~Hz}$. Stiffener spacing: $d=1 \mathrm{~m}$. 
FIG. 9: Propagation zones (lined) and attenuation zones in $(f, k)$ space for plates with different rib spacing: (a) $\mathrm{d}=1 \mathrm{~m},(\mathrm{~b}) \mathrm{d}=1.35 \mathrm{~m}$, (c) $\mathrm{d}=1.5 \mathrm{~m} . \bar{k}^{\mathrm{BF}}=\sqrt{\left(\bar{k}_{x}^{\mathrm{BF}}\right)^{2}+\left(k_{y}^{\mathrm{BF}}\right)^{2}}$. Full line, acoustic wavenumber $k_{0}=2 \pi f / c_{0}$. Black circle on the frequency axis, frequencies showing the propagation zone in the acoustic domain.

FIG. 10: Frequency spectrum of the pressure radiated at $(0,0,10 \mathrm{~m})$ for plates with different rib spacings: (a), d=1 m; (b), d=1.35 m; (c), d=1.5 m. Black circle on the frequency axis, frequencies showing the propagation zone in the acoustic domain ( $\mathrm{dB}$, ref. 1 $\left.\mu \mathrm{Pa} . \mathrm{Hz}^{-0.5}\right)$. 